\title{
Assessment of asthma treatment against SARS CoV-2 by using a computer approach
}

\author{
Halima Hajji ${ }^{1 *}$, Khalil El Khatabi ${ }^{1}$, Hanane Zaki ${ }^{3}$, Fatima En-nahli ${ }^{1}$, Lhossain Hajji ${ }^{2}$, Tahar Lakhlifi ${ }^{1}$, Mohammed Aziz \\ Ajana $^{1 *}$, Mohammed Bouachrine ${ }^{1,3}$. \\ ${ }^{1} 1$ Molecular chemistry and Natural Substances Laboratory, Faculty of Science, University Moulay Ismail, Meknes, Morocco \\ ${ }^{2}$ health, environment, and epigenetics research team, Faculty of Science, University Moulay Ismail, Meknes, Morocco \\ ${ }^{3}$ Superior School of Technology - Khenifra (EST-Khenifra), University of Sultan My Slimane, PB 170, Khenifra 54000 Morocco.
}

\begin{abstract}
The disease caused by the coronavirus is called COVID-19. The degree of infection varies from one person to another. According to the data collected to date, people with asthma and obesity are overrepresented among adults hospitalized for COVID-19. The reason is very simple: COVID-19 is a disease that particularly attacks the respiratory system, including the lungs. This pandemic has led us to return to plants. Modern medicine has found its success thanks to traditional medicine, the effectiveness of which comes from medicinal plants. Currently, in China, many people believe in the miraculous power of plants, boosting their immunity to protect against asthma. Therefore, this work aimed to study components of natural origin that have an anti-asthma effect that can be considered as the panacea against Covid-19, by using the most important method, which is molecular docking. In this research, we performed a molecular docking study on molecules naturally occurring molecules based on the recently crystallized SARS CoV-2 protein (pdb code 7C6S). ADMET prediction was performed for the selected inhibitors. The results of molecular docking and ADMET prediction support the potential of the five selected molecules to be further developed as novel inhibitors for the treatment of SARS CoV-2.
\end{abstract}

\section{INTRODUCTION}

Nowadays, respiratory diseases are a huge public health burden and the currently well-known diseases are asthma and Covid-19. Asthma is a chronic disease that affects humans due to inflammation of the airways in the lungs [1], When the muscle around the bronchi contracts and a lot of phlegm build up in the airways, causing a blockage, it includes symptoms mainly manifested by a cough throughout the day and leads to wheezing. People suffering from asthma may have trouble sleeping, fatigue and poor concentration, dyspnea, These symptoms are most severe at night and are variable over time in frequency and from one person to another [2,3].

At the beginning of the SARS-COV-2 coronavirus pandemic [4], the medical professionals were really afraid of seeing too many asthma patients infected with the virus because, as we know, COVID-19 is caused by a virus that directly affects the lungs, so they share several symptoms. Recently, Chinese studies revealed that there was not a significant proportion of asthma patients infected with the virus [5-7]. On the other hand, there are exceptions for people with asthma if they have relatively severe asthma that is poorly controlled and is also a possible component of Chronic Obstructive Pulmonary Disease (COPD). In these cases, they may be more at risk of having complications from a more severe COVID-19 disease [8].

Furthermore, due to the benefits of traditional Chinese medicine (TCM), which plays an effective role in the treatment of asthma and may also give hope for controlling or helping reduce inflammation in patients with COVID-19 [9], humans have primarily used plants for treatment. However, in this research, we shed light on the effectiveness of polyphenols extracted from Chinese plants which have anti-inflammatory properties that could fight against COVID-19 infection [10,11], from computational studies which focus on the interactions between ligand-receptor by molecular docking method, as well as the toxicity study of these plants from ADMET [12].

\section{Materials and methods}

\subsection{Selection of bioactive polyphenols from medicinal plants}

In this research, we focused on 11 compounds based on polyphenols extracted from different Chinese medicinal plants. Figure 1 shows the structures of the molecules studied as well as the latter were considered to perform the molecular docking study [13].

\footnotetext{
${ }^{*}$ Corresponding author: a.ajanamohammed@ffs.umi.ac.ma ; hal.hajii@edu.umi.ac.ma
} 


\subsection{ADMET prediction}

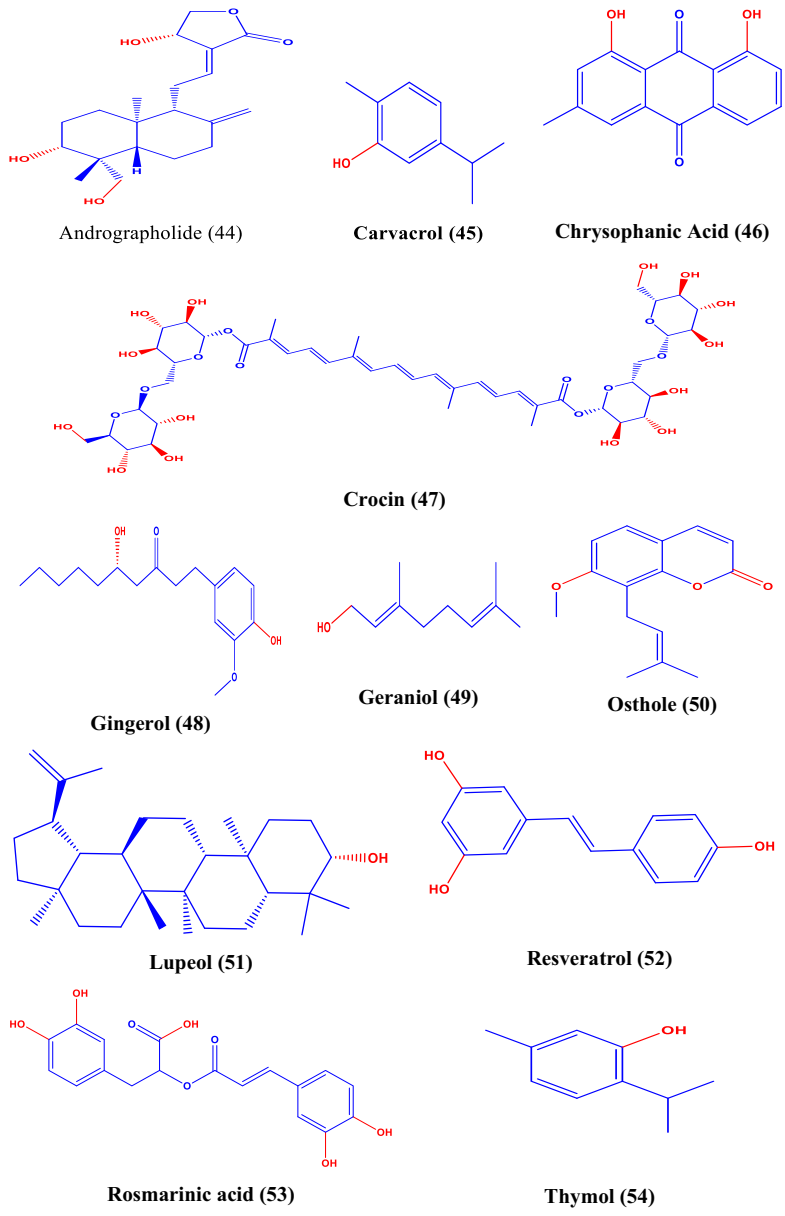

Fig. 1. Chemical structures of 11 selected polyphenols (44-54)

\subsection{Molecular docking}

The main purpose of Molecular Docking is to predict the position of a ligand in its binding pocket [14]. Molecular docking was employed using the Surflex-Dock method in Sybyl X-2.0. The crystal structure 7C6S was provided by the RSCB Protein Data Bank (https://www.rcsb.org/). Using the Discovery Studio 2016 software, all water molecules and the original ligand were removed from the protein, and the binding modes were visualized using PyMOL software $[15,16]$. The preparation steps for docking protocol were used to set up molecular docking with default parameters.

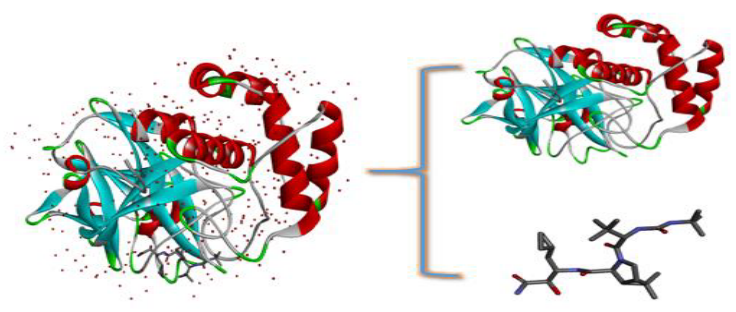

Fig. 2. Crystal structure of SARS CoV-2 main protease (PDB ID: 7C6S), Co-crystallized ligand, and prepared protein for docking
The study of plants becoming drugs in the body over time is called pharmacokinetics. The method of using ADMET type filters (Absorption, Distribution, Metabolism, Elimination) and Toxicology, opens up the possibility of filtering and selecting the molecules according to different parameters and their potential toxicity. Additionally, Lipinski's rules allow rapid identification on a large scale of molecules of a "druglike" nature that are more likely to exhibit bioavailability characteristics [17].

\section{Results and discussion}

\subsection{Molecular docking}

We used the Surflex-dock method in Sybyl to find the different types of interactions that are visualized by using discovery software on 11 molecules of different medicinal plants, and the results are grouped in Table 1. The results show the strong interactions of the plants that can inhibit the SARS-CoV-2. In this docking analysis, we used the protein 7C6S, which has a good resolution: $1.60 \AA$ on these Polyphenols. Furthermore, Met 49, ASP 187, TYR 54, GLY 138, GLU 166, and ARG 188 were discovered to be the main residues of hydrogen bond interactions for Crocin, which is the most active molecule among the studied molecules. We observed that Geraniol made two strong hydrogen bond interactions with THR 190 and ARG 188 residues with small distances of 2.16 and $2.48 \AA$ respectively, and interactions of alkyl and Pi-Alkyl with amino acids HIS 41, MET 49, and MET 165. Similarly, we visualized the interactions in Carvacrol is almost identical to the previous plant, with two hydrogen bond interactions and two interactions between Alkyl and Pi-Alkyl, which is often facilitated by the stabilization of the latter two complexes. For Chrysophanic Acid, we observed two interactions of Hydrogen Bond with THR 190 and ARG188 with distances ranging from 2.14 to $2.59 \AA$ and only a Pi-Alkyl interaction with the residue of ALA 191. Regarding Tymol and Osthole, this study revealed that there are other types of interactions other than the hydrogen bond, an interaction observed between Pi-Lone Pair with THR 190 from Tymol and Pi-Sigma which forms a bond with residue HIS 41. On the other hand, for Osthole, we excluded from our research the components of Gingerol, Resveratrol, Rosmarinic acid, and Andrographolide because of the presence of unfavorable interactions between different regions of these plants.

Table 1. Interactions and key residues (amino acids) for the inhibitors of SARS-CoV2

\subsection{ADMET study}


We performed the in silico ADMET study that aims to eliminate weak or toxic compounds and examine the ability of those that can become a drug by respecting ADMET parameters and Lipinski rules. The following table shows the different parameters of ADMET for molecules that gave good interactions in molecular docking, such as Crocin, Lupeol, Geraniol, Carvacrol, Chrysophanic Acid, Thymol, and Osthole. Through the results, we noticed Crocin has an absorbance value of 0 , indicating that it can't be absorbed by the human intestine, and $\log \mathrm{BB}$ indicates that it is poorly distributed to the brain and does not comply with the five rules of Lipinski Tables 3. Similarly, according to Lipinski's Rule, the parameter $\log \mathrm{P}$ for Lupeol exceeds 5. On the contrary, for the molecules: Geraniol, Carvacrol, Chrysophanic Acid, Thymol and Osthole, these results presented in Table 2 revealed that all these components had very good absorption, which is greater than $70 \%$, and good distribution and excretion and what concerns the results at the level of the AMES test of Toxicity. They indicate that just the molecules of Chrysophanic Acid and Osthole are active as toxic, but the others are not toxic and respect the Lipinski's Rule of Five.

Table 2. The results of the ADMET test of all compounds

Table 3. Lipinski's rule of all compounds in the dataset

\section{Conclusion}

In this work, we focused on the issue of asthma and covid-19. The link between these two diseases is strong because they both attack the respiratory system. That is the reason why we thought this time to turn to traditional Chinese medicine, which pushed us to look for polyphenols that are known in the literature for their effectiveness in asthma and to find compounds that can be used in the management of asthma. For this reason, we carried out a molecular docking study with the main protease of SARS-CoV-2 on 11 polyphenol-based molecules and the results were satisfactory. This study helps us with the analysis of ADMET to propose some plants such as Geraniol, Carvacrol, Chrysophanic Acid, Thymol, and Osthole as a treatment for COVID-19 infections. These plants do not present any risk of toxicity, so they respect Lipinski's Rules. For further study, the synthesis of these compounds and the examination of their in vitro activity against SARS-CoV2 could be of interest.

\section{References}

1. A. Beurnier, E.-M. Jutant, M. Jevnikar, A. Boucly, J. Pichon, M. Preda, M. Frank, J. Laurent, C. Richard, X. Monnet, J. Duranteau, A. Harrois, M.-C. Chaumais, M.F. Bellin, N. Noël, S. Bulifon, X. Jaïs, F. Parent, A.
Seferian, L. Savale, O. Sitbon, D. Montani, and M. Humbert, Eur. Respir. J. 2001875 (2020).

2. M. Morais-Almeida, R. Aguiar, B. Martin, I. J. Ansotegui, M. Ebisawa, L. K. Arruda, M. Caminati, G. W. Canonica, T. Carr, G. Chupp, J. Corren, I. Dávila, H.-S. Park, N. A. Hanania, L. Rosenwasser, M. SánchezBorges, J. C. Virchow, A. Yáñez, J. A. Bernstein, L. Caraballo, Y.-S. Chang, M. Chikhladze, A. Fiocchi, S. N. González-Diaz, L. K. Tanno, M. Levin, J. A. OrtegaMartell, G. Passalacqua, D. B. Peden, P. W. Rouadi, J. L. Sublett, G. W. K. Wong, and E. R. Bleecker, World Allergy Organ. J. 13, 100126 (2020).

3. A. Hughes-Visentin and A. B. M. Paul, Clin. Med. Insights Circ. Respir. Pulm. Med. 14, 117954842096624 (2020).

4. N. Zhu, D. Zhang, W. Wang, X. Li, B. Yang, J. Song, X. Zhao, B. Huang, W. Shi, R. Lu, P. Niu, F. Zhan, X. Ma, D. Wang, W. Xu, G. Wu, G. F. Gao, and W. Tan, N. Engl. J. Med. 382, 727 (2020).

5. World Allergy organization (WAO) https://www.worldallergy.org/COVID-19Information (2020), Accessed 27th Mar 2020.

6. D. M. G. Halpin, R. Faner, O. Sibila, J. R. Badia, and A. Agusti, Lancet Respir. Med. 8, 436 (2020).

7. J. Bedford, D. Enria, J. Giesecke, D. L. Heymann, C. Ihekweazu, G. Kobinger, H. C. Lane, Z. Memish, M. Oh, A. A. Sall, A. Schuchat, K. Ungchusak, and L. H. Wieler, The Lancet 395, 1015 (2020).

8. M. Morais-Almeida, H. Pité, R. Aguiar, I. Ansotegui, and J. Bousquet, Int. Arch. Allergy Immunol. 181, 680 (2020).

9. J. Ren, A.-H. Zhang, and X.-J. Wang, Pharmacol. Res. 155, 104743 (2020).

10. E. Levy, E. Delvin, V. Marcil, and S. Spahis, 61 (n.d.).

11. G. Giovinazzo, C. Gerardi, C. Uberti-Foppa, and L. Lopalco, Molecules 25, 5888 (2020).

12. H. Luo, Y. Gao, J. Zou, S. Zhang, H. Chen, Q. Liu, D. Tan, Y. Han, Y. Zhao, and S. Wang, Chin. Med. 15, 94 (2020).

13. J.-X. Liu, Y. Zhang, H.-Y. Yuan, and J. Liang, J. Ethnopharmacol. 269, 113558 (2021).

14. K. El Khatabi, İ. Aanouz, R. El-MerniSsi, A. Khaldan, M. A. Ajana, M. Bouachrine, and T. Lakhlifi, J. Turk. Chem. Soc. Sect. Chem. 469 (2020). 
15. H. Hajji, F. En-Nahli, O. Abdessadak, K. E. Khatabi, M. A. Ajana, T. Lakhlifi, and M. Bouachrine, 12, 20 (2021).

16. K. El Khatabi, I. Aanouz, R. El-mernissi, A. Khaldan, M. A. Ajana, M. Bouachrine, and T. Lakhlifi, Orbital Electron. J. Chem. 12, 172 (2020).
17. A. Khaldan, S. Bouamrane, F. En-Nahli, R. ElMernissi, K. El Khatabi, R. Hmamouchi, H. Maghat, M. A. Ajana, A. Sbai, M. Bouachrine, and T. Lakhlifi, Heliyon 7, e06603 (2021).

\section{Tables}

Table 1. Interactions and key residues (amino acids) for the inhibitor of SARS-CoV2

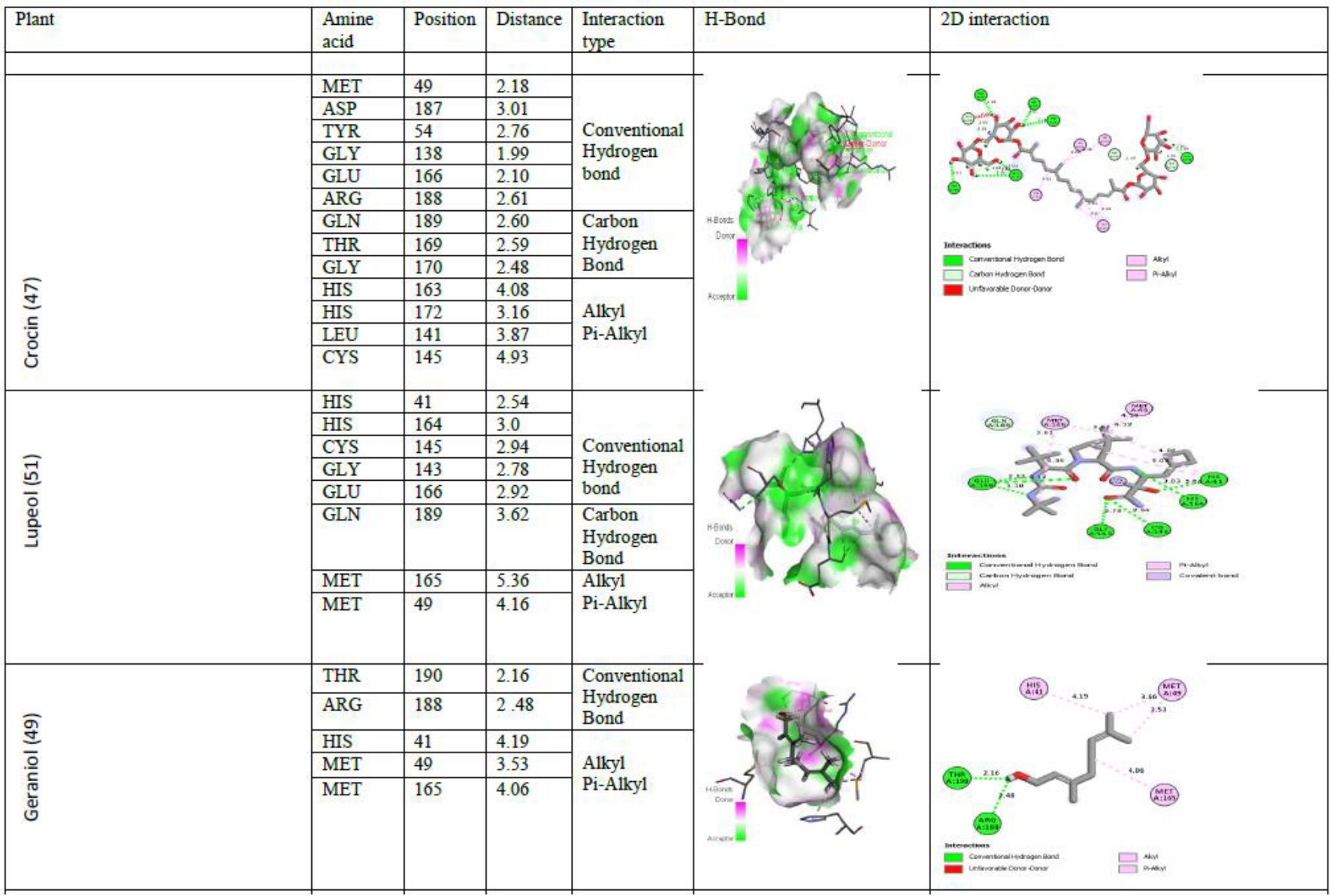




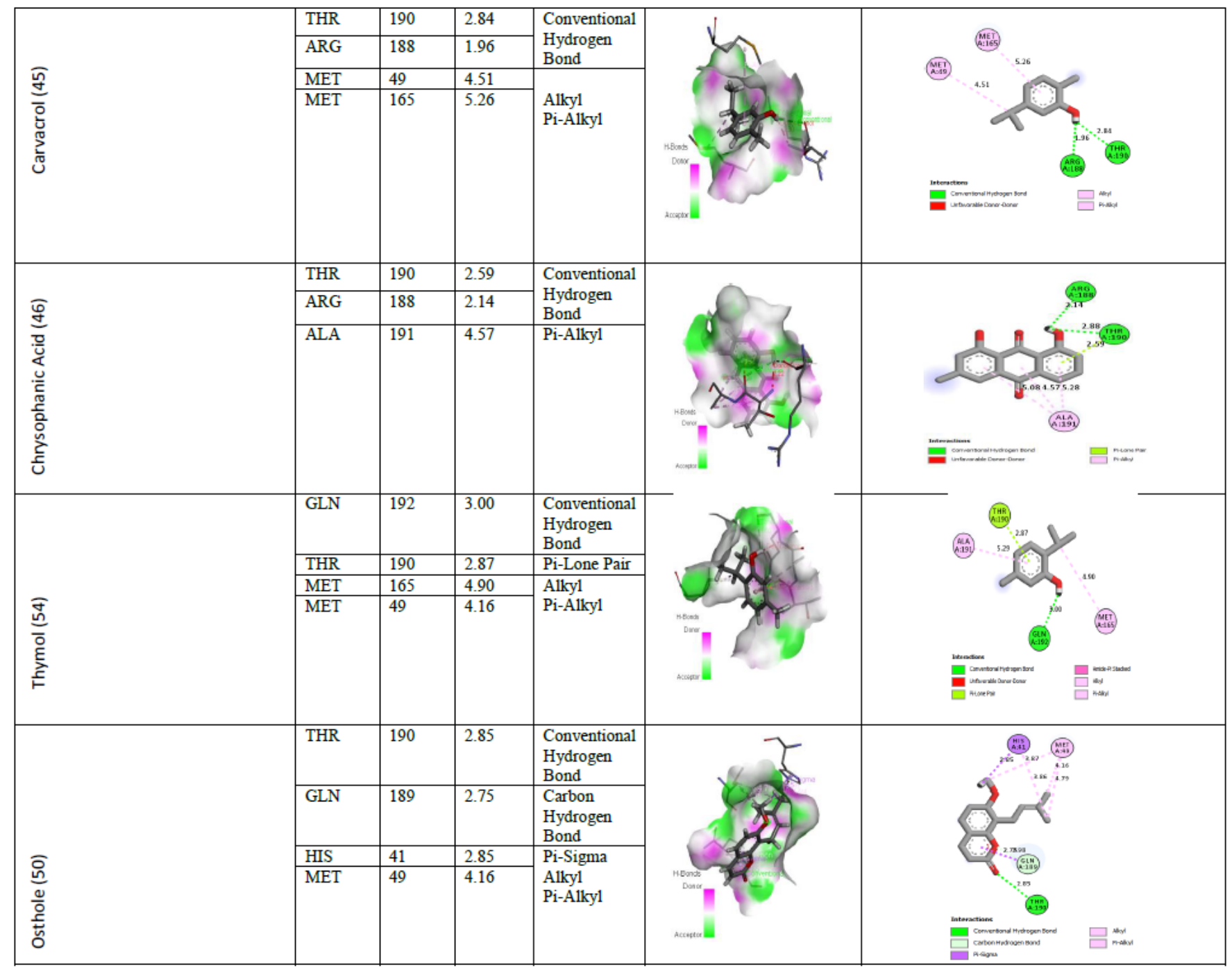

Table 2. The results of the ADMET test of all compounds

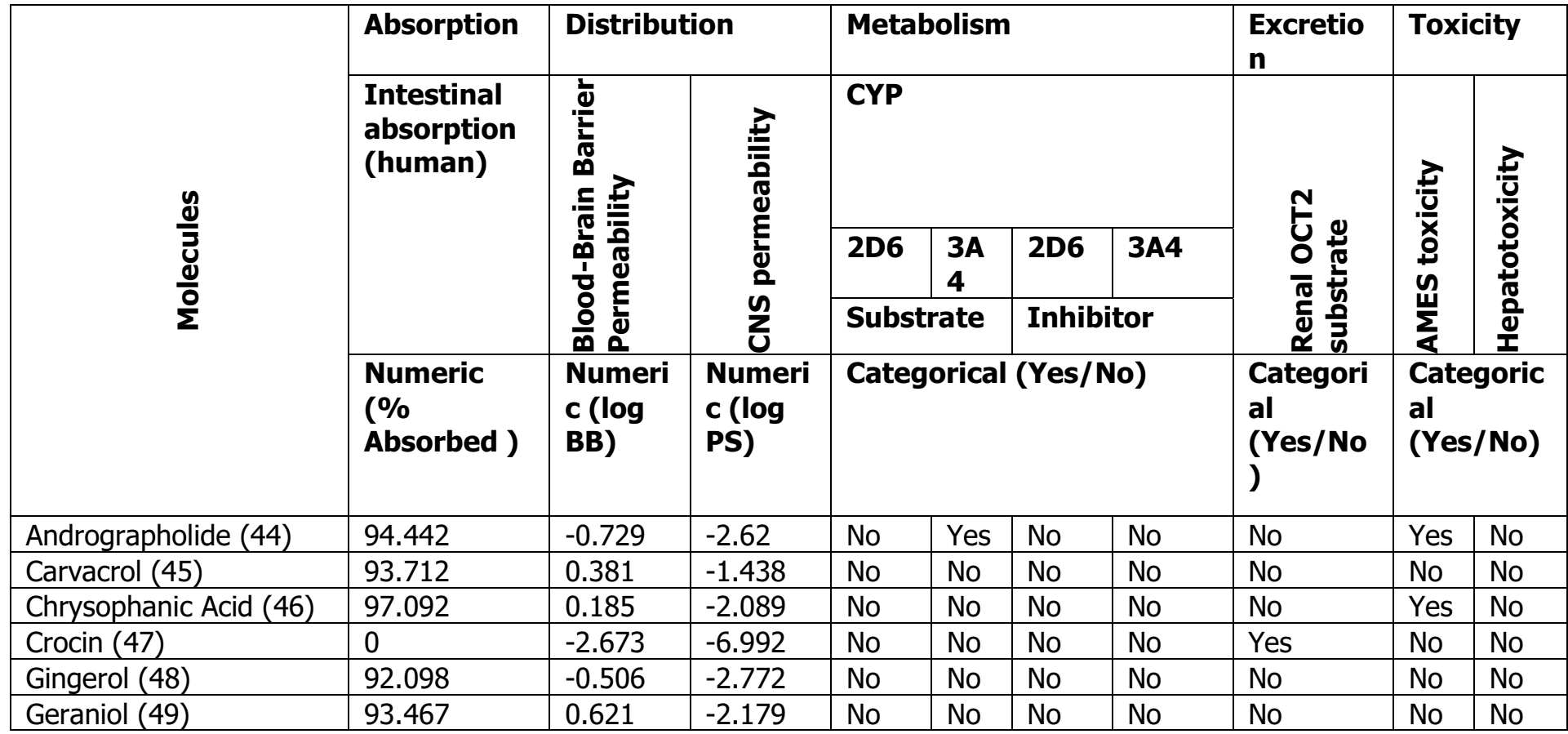




\begin{tabular}{|l|l|l|l|l|l|l|l|l|l|l|}
\hline Osthole (50) & 96.049 & 0.162 & -2.183 & No & Yes & No & Yes & No & Yes & No \\
\hline Lupeol (51) & 99.868 & 0.739 & -1.35 & No & Yes & No & No & No & No & No \\
\hline Resveratrol (52) & 89.057 & -0.041 & -2.098 & No & No & Yes & Yes & No & No & No \\
\hline Rosmarinic acid (53) & 53.567 & -1.547 & -3.567 & No & No & No & No & No & No & No \\
\hline Thymol (54) & 93.24 & 0.336 & -1.349 & No & No & No & No & No & No & No \\
\hline
\end{tabular}

Table 3. Lipinski's rule of all compounds in the dataset

\begin{tabular}{llllll}
\hline Compounds & Molecular weight (g/mol) & LogP & $\begin{array}{l}\text { H-bond } \\
\text { acceptors }\end{array}$ & $\begin{array}{l}\text { H-bond } \\
\text { donors }\end{array}$ & $\begin{array}{l}\text { Rotatable } \\
\text { bonds }\end{array}$ \\
\hline Andrographolide (44) & 350.455 & 1.96 & 5 & 3 & 3 \\
Carvacrol (45) & 150.221 & 2.824 & 1 & 1 & 1 \\
Chrysophanic Acid (46) & 254.241 & 2.181 & 4 & 2 & 0 \\
Crocin (47) & 976.972 & -5.225 & 24 & 14 & 18 \\
Gingerol (48) & 294.391 & 3.233 & 4 & 2 & 10 \\
Geraniol (49) & 154.253 & 2.671 & 1 & 1 & 4 \\
Osthole (50) & 244.29 & 3.310 & 3 & 0 & 3 \\
Lupeol (51) & 426.729 & 8.024 & 1 & 1 & 1 \\
Resveratrol (52) & 228.247 & 2 & 3 & 3 & 2 \\
Rosmarinic acid (53) & 360.318 & 1.761 & 7 & 5 & 6 \\
Thymol (54) & 150.221 & 2.824 & 1 & 1 & 1 \\
Reference ligand & 150 to 500 & -0.7 to +5.0 & $\leq 10$ & $\leq 5$ & $\leq 9$ \\
\hline
\end{tabular}

